\title{
Optimum parameters for the measurement of cortical coupling
}

\author{
AKIHIRO YAGI, LEKH BALI, and ENOCH CALLAWAY \\ Langley Porter Neuropsychiatric Institute, University of California Medical Center \\ San Francisco, California 94143
}

\begin{abstract}
Electrical coupling between areas on the human scalp can be estimated using information transmission as a measure of contingency. Such a measure of coupling changes as a subject's cognitive operations change. Based on a study of six subjects, detection of cognitively induced changes in coupling is optimum when contingencies are computed from EEG epochs that are about $1.6 \mathrm{sec}$ in duration, sampled every $4 \mathrm{msec}$ with each sample classified as to polarity and first difference.
\end{abstract}

Callaway and Harris (1974) reported on a simple measure of cortical coupling which changes significantly with changes in the subject's cognitive processes. This present study was designed to determine those parameters of measurement that best reveal changes in coupling when cognitive processes change.

Three parameters are examined. These were (1) lead placement, (2) method of coding sample EEG voltages, and (3) window size.

The earlier study examined coupling of a posterior lead to central leads, so in the present study we examined coupling of an occipital lead to frontal and parietal leads to see what this might disclose.

The early study coded EEG samples on the basis of polarity and first derivative. We had assumed that these would be the more or less instantaneous qualities of potentials that nerve membranes might sense. To give a more empirical justification, coding according to polarity alone and coding according to polarity with first and second derivatives were added to the previously used polarity and first-derivative coding.

Finally, we wished to use a range of window sizes both (a) to permit selection of the best window size for future work and (b) to determine whether rules governing the relationship between patterns of electrical potentials in two functionally related cortical areas are relatively invariant, or whether they change from time to time. Elul (1972) has given evidence that these "rules" hold only for intervals on the order of $1 \mathrm{sec}$. If the rules are invariant, large windows would give the best estimates of coupling. If the rules change, large windows would encompass several changes of rules and these transient meaningful patterns of relationship would be obscured.

This work was supported by Office of Naval Research No. N00014-69-A-0200-2007, General Research Support Grant FR-05550-11 and a special fellowship from the Government of Japan. The first author's present address is Industrial Products Research Institute, 2-12, 4-chome, Shimomaruko, Ohtaku, Tokyo 144 Japan. Please send reprint requests to Enoch Callaway.
This study also provides a partial test of two alternative explanations for changes in cortical coupling with changes in cognitive activity. These alternatives are (1) an increase in the measure of coupling could reflect an increase in the coincidence of a specific electrical event at one area with a specific event at the other, and/or (2) an increase in the measure of coupling could reflect a decrease in the independent signals arising from the two areas under study, relative to a common signal that appears at both areas because of passive (electrotonic) conduction.

\section{METHOD}

\section{Subjects}

Subjects were six right-handed adults who gave no histories suggesting left-handedness.

\section{Recording}

Electrodes were placed on left and right frontal (F3, F4), left and right parietal (P3, P4), and midline occiput (Oz). Each was referred to vertex (Cz). Five channels of EEGs were amplified with Beckman amplifiers at a low-frequency time constant of $.3 \mathrm{sec}$ and a high-frequency cutoff of $30 \mathrm{~Hz}$. EEGs were digitized every $4 \mathrm{msec}$ on-line with a PDP-15 computer using a 12-bit A/D conversion and recorded on Dectape. Recordings were made while the subject performed a series of tasks during each of which $86 \mathrm{sec}$ of EEG was recorded.

\section{Procedure}

Subjects were seated comfortably at a small table in a sound-attenuated room after the attachment of electrodes. There were five experimental conditions as follows: (1) Eyes open: The subject was instructed to relax with his eyes open. (2) Eyes closed: The subject was instructed to relax with his eyes closed. (3) Scrabble (right hand): Seven small cardboard squares, each printed with a letter of the alphabet, were presented to the subject, and the subject was asked to make as many words as possible using only his right hand. He was asked not to move his left hand and to keep his eyes as stable as possible while performing a task. (4) Scrabble (left hand): This was the same as above except that the operating hand was changed from right to left. (5) Block design (right hand): A block design task of the Wechsler Adult Intelligence Scale was given to the subject. This requires that the subject construct patterns with nine colored blocks to match a series of models. The subject was instructed to use only his right hand and to keep 
his eyes as stable as possible. (6) Block design (left hand): This was the same as above except that the subject was permitted to use only his left hand.

The order of the tasks from 3 to 6 was randomized for each subject. Additional problems were given to the subject as needed so as to maintain each task long enough for the required recording interval.

\section{Analysis of Data}

The digital data which had been recorded on-line on magnetic tape (Dectape) was processed off-line on the same PDP-15 computer. As noted above, five derivations were used. These were $\mathrm{F} 3, \mathrm{P} 3, \mathrm{P} 4, \mathrm{~F} 4$, and $\mathrm{Oz}$, all referred to $\mathrm{Cz}$. Our intention was to investigate coupling of each of the frontal $(\mathrm{F} 3-\mathrm{Cz}, \mathrm{F} 4-\mathrm{Cz})$ and parietals $(\mathrm{P} 3-\mathrm{Cz}, \mathrm{P} 4-\mathrm{Cz})$ channels to the occiput $(\mathrm{Oz}-\mathrm{Cz})$.

In addition to the comparison between the four pairs of derivations, there were two other parameters. These were method of coding and window size. Three different methods of coding were used to compute the degree of coupling. The first method of coding used polarity only (zero derivative). Each of two EEGs sampled every $4 \mathrm{msec}$ was classified as to whether polarity was positive or negative, and the contingencies were tallied into a 2 by 2 table. The second method used polarity and first difference (one derivative). Each of the samples was classified as to polarity and whether it was greater than or less than the preceding sample from the same channel. Thus, the first difference was used to estimate first derivative. In this way each sample was classified in one of four categories and the contingencies tallied into a 4 by 4 table. The third method used polarity and first and second differences (two derivatives). Each sample was classified as to polarity, as to first difference, and as to whether this first difference was greater or less than the preceding first difference. Thus, the second difference was used to estimate the second derivative. In this way, each sample was classified into one of eight categories and the contingencies tallied into an 8 by 8 table.

Window size refers to the number of samples used in making up each contingency table. This was the third parameter. In this study five window sizes were used. These were 100, 200, 400,800 , and 1600 samples. As the total length of the EEG for each task was fixed at $86 \mathrm{sec}$ and samples were taken every $4 \mathrm{msec}$, the total number of samples per channel for each task was 21,504 . Thus the number of contingencies that was computed and averaged for each window size was different. They were, respectively, 215, 107, 53, 26, and 13 .

The two samples collected just prior to the start of each window were used to compute the first and second differences for the tirst sample in the window, and the average over the window was used to determine the baseline against which polarity was measured.

The Shannon-Weaver information transmission (or uncertainty reduction) statistic $\mathrm{T}(\mathrm{xy})$ (Garner, 1962; Shannon \& Weaver, 1949) was used to measure contingency (coupling). In a contingency table each cell represents the total coincidence of a specific classification of an EEG sample in channel X with the specific classification of an EEG sample from channel Y.

The most familiar nonparametric contingency statistic is chi square. $T(x y)$ can be considered essentially analogous to chi square computed using log average frequencies so that addition rather than multiplication can be used to compute expected frequency.

Let a single channel $\mathrm{X}$ be sampled at $\mathrm{K}$ points in time. Classify each sample into one of $\mathrm{C}$ classes. If $\mathrm{Ni}$ is the number of samples in class $i$, then the estimated probability (P) of a sample in channel $\mathrm{X}$ belonging to class $\mathrm{i}$ will be:

$$
P\left(x_{i}\right)=\frac{N i}{K}
$$

and the uncertainty $(\mathrm{Hx})$ of channel $\mathrm{X}$ will be:

$$
H(x)=\underset{i=1}{C} P\left(x_{i}\right) \log _{2} P\left(x_{i}\right)
$$

and for two channels (X and $\mathrm{Y}$ ), sampled and classified simultaneously into $\mathrm{i}$ and $\mathrm{j}$ classifications:

$$
H(x, y)=\underset{i=1}{C} \sum_{j=1}^{C} P\left(x_{i}, y_{j}\right) \log _{2} P\left(x_{i}, y_{j}\right)
$$

Reduction in uncertainty about $\mathrm{X}$ given $\mathrm{Y}$ or vice versa is known as information transmission and is given by:

$$
T(x y)=H x+H y-H(x, y)
$$

This measure has no theoretical advantage over chi square or, for that matter, over an autocorrelation function. It does, of course, have an advantage over an ordinary cross-correlation, since sine waves $90 \mathrm{deg}$ out of phase give .0 correlation with the usual cross-product method but show high contingency by this method. In practice, since the range of $P$ is finite and defined by the window size (number of samples), all possible $\left(\mathrm{P} \log _{2} \mathrm{P}\right)$ values can be computed in advance and the entire $\mathrm{T}(\mathrm{xy})$ calculation carried out by table look-up and by addition in fixed-point arithmetic.

For example, if a window contains $\mathrm{K}$ samples, the possible $P$ (estimated probability values) will range as follows:

$$
\frac{0}{\mathrm{~K}}, \frac{1}{\mathrm{~K}}, \frac{2}{\mathrm{~K}}, \ldots \frac{\mathrm{K}}{\mathrm{K}}
$$

Thus, for a window of $K$ samples a table of $(-P \log P)$ values containing only $\mathrm{K}+1$ entries will cover all possible situations. Then computation of $\mathrm{H}(\mathrm{x})$ for channel $\mathrm{X}$ divided into $\mathrm{C}$ classes can proceed as follows: (a) Set $\mathrm{i}=1$. (b) Look up Xi. (c) Look up value of $-\mathbf{P}(\mathrm{xi}) \log (\mathrm{Pxi})$ from the table. (d) Accumulate value obtained in $H(x)$. (e) Increment $i$ and start again at Step $b$ until all $\mathrm{C}$ classes of $\mathrm{Xi}$ have been treated.

\section{RESULTS}

The primary purpose of this study was to determine the parameters that would optimize the detection of change in coupling between cortical areas produced by changes in cognitive operations. The two tasks, each performed both with right and with left hand, were selected with the idea that "scrabble" and right hand would call on the left hemisphere with increased left hemisphere occiput coupling, while "block design" and left hand would call on the right hemisphere with increased right hemisphere occiput coupling. In the previous study (Callaway \& Harris, 1974), we noted that people do not always show the expected changes in cortical coupling without some practice and instruction. This is not surprising since verbal strategies may be employed to solve apparently nonverbal tasks and vice versa. In this study, rather than train each subject as in our previous study, we instead allowed the subjects to perform as they pleased and focused attention on the significant changes as they occurred.

Figures 1 and 2 summarize part of the data from one subject and serve as an example for the total study. Figure 1 summarizes data for only the 400 sample 


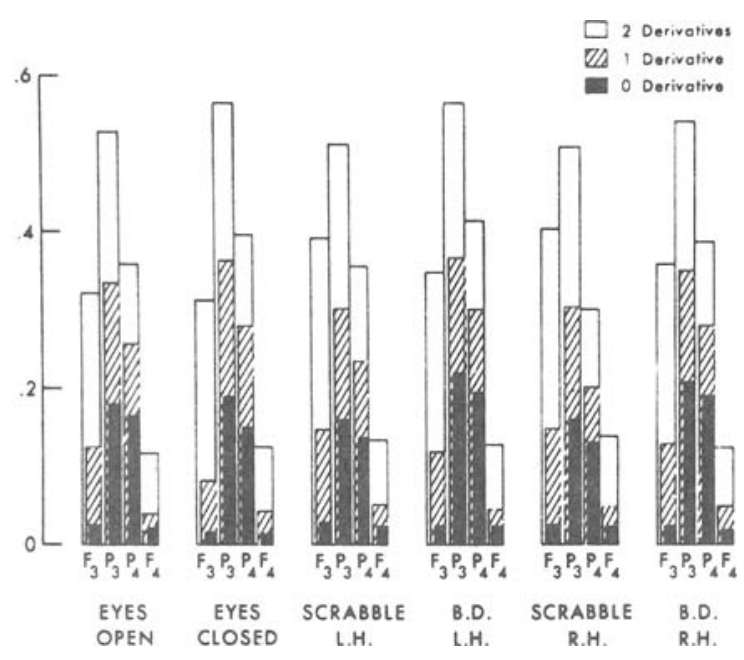

Figure 1. Data from a single subject showing coupling $T(x y)$ between occiput $(\mathrm{Oz})$ and leads shown (all referred to $\mathrm{Cz}$ ) for six task conditions and three coding systems. Only 400 sample window data are given.

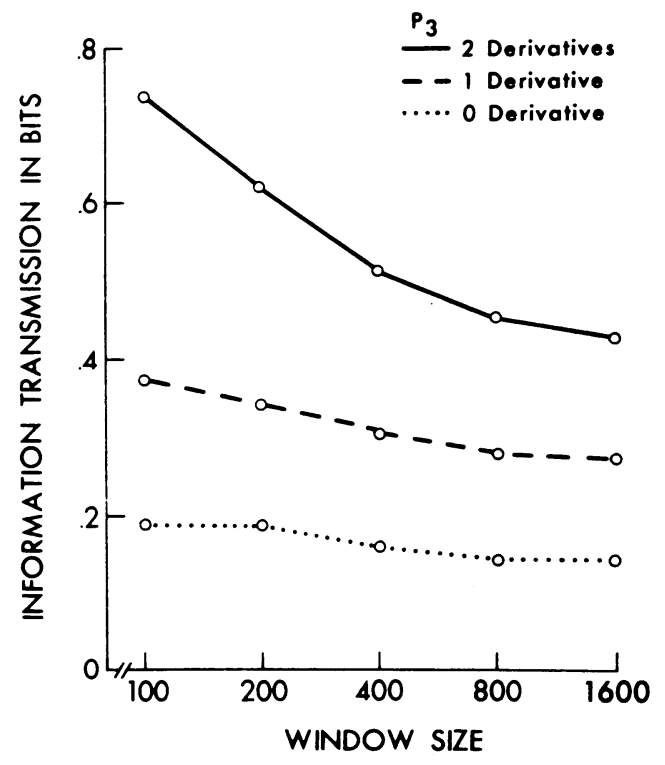

Figure 2. Data from the same subject as in Figure 1 showing transmission between $\mathrm{Oz} \cdot \mathrm{Cz}$ and $\mathrm{P3}-\mathrm{Cz}$ for three coding task conditions and five window sizes.

window size but for all other parameters. Figure 2 summarizes data for all window sizes and for all orders of coding but only for "scrabble" left hand and F3-Cz coupled to $\mathrm{Oz}-\mathrm{Cz}$. They illustrate certain general findings. First, Figure 1 shows that occiput-parietal (P3, P4) couplings are larger than occiput-frontal (F3, F4) couplings. Figure 1 also shows, as would be expected, transmission is largest for coding according to polarity and two derivatives (two-derivative, 3-bit maximum), next for polarity and one derivative (onederivative, 2-bit maximum), and least for polarity alone (zero-derivative, 1-bit maximum). This point is further illustrated in Figure 2, which also shows that transmission values fall as window size increases.

To determine the most advantageous parameters for detecting significant changes, a series of $t$ tests were made. Each transmission during "scrabble" was compared with that transmission during "block design" that was from the same subject and had the same parameters. Thus, for six subjects, four derivatives, two hands, five window sizes, and three coding classifications, there were 720 such pairs to compare. Degrees of freedom for each of the $t$ tests depended on window size, as noted above. The results are summarized in Table 1. One hundred and seventy-one $t$ tests were significant at $\mathrm{P}>.05$. This is slightly less than one in five. However, on examining the table, we see that zero-derivative code was relatively ineffective and yielded only one in eight significant changes, while the one-derivative code and two-derivative code were more effective and yielded one in four and one in three, respectively. Window size was also an important parameter, with 400 samples being superior both in numbers of significant ts and in numbers of "scrabbleblock" transmission changes in the expected direction. No lead was clearly superior to any other lead in revealing significant changes although frontals fared better than parietals.

Thus, we conclude that the window size of 400 samples $(1.6 \mathrm{sec})$ and the one-derivative $(2$-bit) or two-derivative (3-bit) code were best. Leads remain to be investigated more extensively. Since the 3-bit code is four times as expensive computationally as the 2-bit code, the 2-bit code seems the better choice.

Using this information, we made left- and righthand comparisons using one derivative and 400

Table 1

Distribution of Significant $t$ Tests for Differences in Information Transmission

\begin{tabular}{lccccccc}
\hline \multicolumn{8}{c}{ Information Transmission } \\
\multicolumn{1}{c}{} & \multicolumn{7}{c}{ Window (Sample Size) } \\
Coding & Leads & 100 & 200 & 400 & 800 & 1600 \\
\hline Zero & $\mathrm{F} 3$ & 0 & 0 & 0 & 0 & 0 & 0 \\
Derivative & $\mathrm{P} 3$ & $0 / 10$ & $0 / 1$ & $0 / 2$ & $0 / 2$ & $0 / 3$ & $0 / 2$ \\
$16 / 15$ & $\mathrm{P} 4$ & $12 / 0$ & $2 / 0$ & $2 / 0$ & $4 / 0$ & $2 / 0$ & $2 / 0$ \\
& $\mathrm{~F} 4$ & $4 / 5$ & $0 / 1$ & $2 / 0$ & $2 / 2$ & $0 / 2$ & 0 \\
One & $\mathrm{F} 3$ & $14 / 5$ & $3 / 1$ & $3 / 1$ & $3 / 1$ & $2 / 1$ & $3 / 1$ \\
Derivative & $\mathrm{P} 3$ & $5 / 8$ & $1 / 1$ & $1 / 1$ & $1 / 2$ & $1 / 2$ & $1 / 2$ \\
40/23 & $\mathrm{P} 4$ & $11 / 3$ & $2 / 0$ & $2 / 1$ & $3 / 0$ & $2 / 1$ & $2 / 1$ \\
& $\mathrm{~F} 4$ & $10 / 7$ & $1 / 1$ & $2 / 1$ & $4 / 2$ & $1 / 2$ & $2 / 1$ \\
Two & $\mathrm{F} 3$ & $16 / 11$ & $4 / 1$ & $3 / 1$ & $3 / 3$ & $3 / 3$ & $3 / 3$ \\
Derivative & $\mathrm{P} 3$ & $10 / 7$ & $2 / 1$ & $2 / 1$ & $2 / 2$ & $2 / 2$ & $2 / 1$ \\
$45 / 32$ & $\mathrm{P} 4$ & $8 / 7$ & $1 / 2$ & $2 / 1$ & $3 / 1$ & $2 / 1$ & $0 / 2$ \\
& $\mathrm{~F} 4$ & $11 / 7$ & $1 / 2$ & $2 / 1$ & $4 / 1$ & $2 / 1$ & $2 / 2$ \\
& & & $17 / 11$ & $21 / 10$ & $29 / 16$ & $17 / 18$ & $17 / 15$ \\
\hline
\end{tabular}

Note-Each cell covers 12 pairs /six subject, left hand by right hand). Values above the line are the number of "expected" ts, i.e., scrabble > block for F3 and P3, block > scrabble for F4 and P4. Those below the line are of the opposite sign. 
sample window. Using the same convention as in Table 1, the results are shown in Table 2 . Here there are 11 significant left-hand/right-hand comparisons as contrasted with 16 significant scrabble-block comparisons for the same code and window size. Of these, only $36 \%$ of the significant left-hand/right-hand comparisons were in the expected direction, as compared to $71 \%$ of the scrabble-block comparisons. Why hand had such little and inconsistent effect on transmission measures remains to be determined.

This experiment was not designed to provide another demonstration that in right-handed subjects scrabble is more likely to increase coupling over the left hemisphere than block design, while block design is more likely to increase coupling over the right hemisphere than scrabble. The tally of significant ts most economically summarizes our mass of data for the purposes of selecting optimum parameters. Nevertheless, we have also computed a more conventional statistical test of changes in coupling measures consequent to task performance. We took the data obtained with a 400 sample window where each coupling value was the mean of 53 separate computations. For each subject we computed $t$ values for the differences (mean coupling during scrabble minus mean coupling during block design). The four leads and two hands provide eight sets of data for all six subjects. The one-tailed probabilities of the six $t$ values in each of the eight sets were combined so that the distribution could be tested by chi square with 12 df (Weiner, 1971). The results are summarized in Table 3.

The values above the lines are computed on the basis of one-tailed probabilities given the expectation that scrabble should produce greater coupling than blocks over the left hemisphere and that blocks should produce greater couplings than scrabble over the left hemisphere. The values below the line are similar computations performed with the opposite (or expected) hypothesis. Chi squares for the .05 and .01 levels of confidences are given in the table. Examining the table shows that six of the eight chi squares using the expected hypothesis are significant at better than the .05 level of confidence, whereas three of the eight chi squares using the unexpected hypothesis are significant. In other words, there is a highly significant effect of task, but one that interacts strongly with individual differences and lead positions. There is indeed a tendency for unpracticed and uninstructed subjects to show more right-sided coupling with blocks and left-sided coupling with scrabble, but significant changes in the opposite direction also occur. Further examination shows that larger and more significant effects in the expected direction occur over the right hemisphere when the tasks are performed with the right hand, and over the left hemisphere when tasks are performed with the left hand. One might speculate that the operations involved in controlling the hand
Table 2

t Tests Comparing Left-Hand and Right-Hand Pairs

\begin{tabular}{lllll}
\hline & F3 & P3 & P4 & F4 \\
One Derivative & $0 / 1$ & $1 / 3$ & $1 / 2$ & $2 / 1$ \\
\hline
\end{tabular}

Note-As in Table 1, numbers above the lines are "expected": Right hand $>$ left hand for F3 and P3; left hand > right hand for $P 4$ and F4.

Table 3

Combined One-Tailed Probabilities from Sets of Six $t$ Tests (Weiner, 1972)

Left Hemisphere $\frac{2}{2}$

Note-Values above the lines are "expected" (scrabble > blocks for left hemisphere, blocks > scrabble for right hemisphere). Values below the lines are the opposite or "unexpected." ${ }^{*} X .95(12)=21.0 \quad X .99(12)=26.2$

obscure changes in coupling that result from differences in cognitive operations.

\section{DISCUSSION}

The study was designed primarily to provide an empirical foundation for selecting parameters in subsequent investigations.

Lead position is obviously a major factor. Larger occiput-to-parietal couplings are the rule. This may reflect appearance of passively conducted alpha waves at the two electrodes. Large differences from individual to individual make other inferences hazardous at this point.

The use of polarity and one derivative seems most efficient. Whether or not the use of 2-bit derivative conveys any advantage (regardless of cost) remains a question, because the use of first difference to estimate first derivative sacrificed some of the degrees of freedom inherent in the 2-bit coding scheme. This will be discussed later in the explanation of Figure 3.

A window of $1.6 \mathrm{sec}$ provides the most significant $t$ values. Smaller and larger windows are less effective. The fact that large windows are less effective suggests that more or less invariant patterns of relationship do not account for the changes in coupling that occur with changes in cognitive function. The U-shaped relationship between window size and $t$ values does not seem to be a statistical artifact. The statistic $t$ is a function of the standard deviation of the mean. If changing window size changes standard deviation of the mean, such an effect could account for our finding. Figure 3 shows coupling computed between 


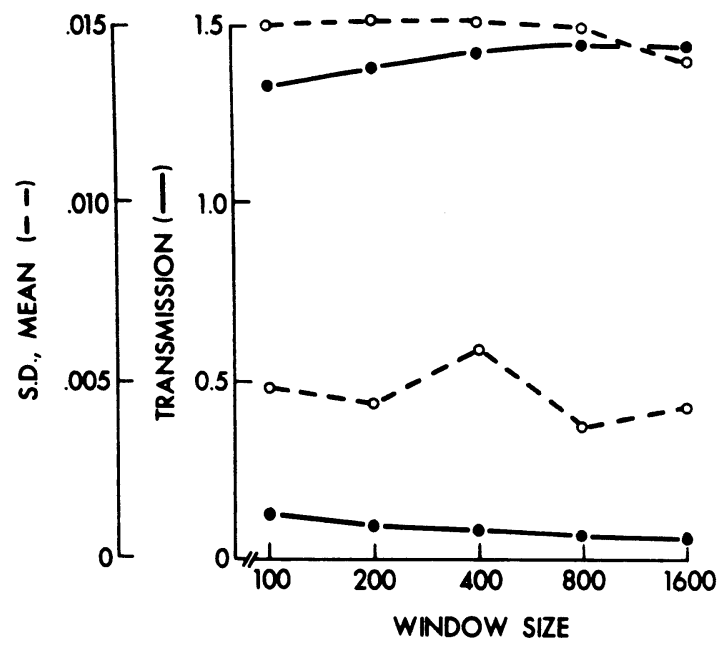

Figure 3. Transmission between signal and its inverse (upper lines) and two unrelated signals (lower lines). Solid lines are mean transmissions using 2-bit (polarity plus one derivative) coding. Dashed lines indicate standard deviations of means plotted on an expanded scale. Changes in standard deviations of the mean are not significant.

the EEGs of two different subjects (no coupling) and coupling computed between the EEG of a given subject and that same EEG simply reversed in polarity. Coding was for polarity and one derivative.

It can be seen that with increasing window size transmission falls monotonically for the uncoupled signals and approaches a value of about 1.5 bits monotonically for the closely coupled signals. Since $T(x y)$ is based on estimated probability, the smaller the sample (window), the greater the random error encountered in estimating probability from frequency. This explains why unrelated signals fail to show zero coupling at the smaller window sizes. At large windows, the existence of similar frequencies in both EEG channels and perhaps some common $60-\mathrm{Hz}$ noise in the $A / D$ section of the computer may account for the small remaining transmission. Variance of transmission is also a function of mean transmission. This suggests that $\log$ transformation might be appropriate for more elegant work, but the standard deviation of the mean is not appreciably influenced by sample size. Thus, the peaking of $t$ values with a window of approximately $1.6 \mathrm{sec}$ probably reflects a real change in coupling that is detected best with a brief window and is obscured if one accumulates the measure of coupling over a longer period, during which the rules for electrical coupling change.

In Figure 3 coupled signals approach a transmission of 1.5 bits rather than the predicted 2 bits. This is due to (a) different amplifier noise for the two channels, (b) digital definition of polarity, (c) sampling lag from channel to channel, and (d) bias produced by using first difference to estimate first derivative. This last point requires some comment. Let $\mathrm{V}(\mathrm{i})$ be a sample at times $\mathrm{i}$ and $\mathrm{V}(\mathrm{i}-1)$ be the preceding sample. We took the sign of $\mathrm{V}(\mathrm{i})$ as polarity and the sign of $\mathrm{V}(\mathrm{i})-\mathrm{V}(\mathrm{i}-1)$ as first derivative. However, when a wave has past its peak and the true first derivative corresponding to $\mathrm{V}(\mathrm{i})$ is negative, the sign $\mathrm{V}(\mathrm{i})$ - V(i - 1) may still be positive. This lag causes an excess of positive polarity/positive firstderivative cases and a like excess of negative polarity/ negative first-derivative cases, at the expense of the remaining two classes. The higher the signal frequency, the greater this bias until signal frequency exceeds one-half the sampling figures. In subsequent work we have corrected this by computing polarity as sign of $\mathrm{V}(\mathrm{i})+\mathrm{V}(\mathrm{i}-2)$ and first difference as the sign of $\mathrm{V}(\mathrm{i})-$ $\mathrm{V}(\mathrm{i}-1)$.

Finally, re-examining Table 1 provides additional support for the contention that a window of about $1.6 \mathrm{sec}$ optimizes the detection of changes in coupling due to changes in cognitive functioning. In each cell two values are given, divided by a slanted line. Those above the line are the number of significant changes in the expected direction (e.g., increases in left coupling with scrabble and increases in right coupling with blocks). The number of predicted significant coupling differences increases to a peak at the window size of 400 and then falls with larger window sizes. On the other hand, the number of unpredicted coupling differences increases as window size increases to 400 samples but then remains high and does not show any subsequent drop, suggesting that the unexpected coupling differences might indeed reflect changes in common signal contamination rather than changes in functional relationship.

The coupling measure was designed to detect the coincidences of one state in one channel with one state in another channel while setting as few constraints as possible on what these states must be. We suspect that for brief periods when two cortical areas join in some computation, then some increase in certain coincidences of electrical patterns will occur. However, the relative increase of signal passively conducted to the two recording sites would also produce an indistinguishable increase in coupling between the two sites by our measure.

In the Callaway and Harris (1974) study, the measures of coupling were taken between posterior scalp (Cz-P2) and left central (Cz-C3) and between posterior scalp and right central (Cz-C4) using 400 sample window and polarity and first difference. Using reading as one task and looking at a picture as another, we found significant changes in coupling with change in task and also noted that most subjects were able with some instruction to show more right coupling when looking at a picture and left coupling when reading a book.

Although it is plausible to consider these results as the consequence of a shift from apositional to propositional mode (Bogen, 1969), there remains the alternative explanation. Change in coupling might not 
reflect a change in common electrical signals but rather the effect of gross changes in total EEG. For example, consider two hypothetical derivations which we will call A-C and B-C, these being considered the differences between signals $A, B$, and $C$, which would be seen if the various leads were referred to a hypothetical inactive reference. If $A$ and $B$ are independent and of high amplitude, then $C$ would contribute little and coupling would be small. If, however, A and B were suppressed, $C$ would then make more of a contribution to both A-C and B-C with an apparent increase in coupling.

In our first study, nonvisual tasks that were presumably left hemisphere (eyes closed, composing a letter) and right hemisphere (eyes closed, listening to music) were included to test this. With eyes closed, EEG suppression should occur over the used hemisphere (Galin \& Ornstein, 1972), but visual cortex coupling to the used hemisphere (since eyes were closed) should not be increased. Eyes-closed differences were found but were not consistent. Thus, the data supported the cortical coupling hypothesis as opposed to the EEG suppression hypothesis, but only weakly.

Several other approaches are possible. For example, the application of three-dimensional transmission measures (McGill, 1954) will allow computation of partial transmission (e.g., coupling of A-C to B-C with D-C effects partialed out). Before undertaking this more complex and expensive three-dimensional calculation, however, we needed to answer the parametric question addressed in this study.

Nevertheless, the data from this study also shed light on the question as to whether or not coupling simply reflects common signal contamination. If there were a common contaminating signal, phase relationships at two derivations would be invariant and the longer the window, the better would be the estimate of coupling. If, however, a more dynamic coupling existed, we might expect transient phase relationships. Elul (1972) has shown that periodic high correlations occur between slow waves and the spike frequency of single neurons, but these periods of correlation last only about a second. Thus, there is some independent evidence to support the idea of transient relationships between cortical areas when information is being transferred. If this were the case, there would be a degradation in the estimation of coupling when the window spanned a greater time than that over which a relationship was stable. The fact that the maximum number of significant tests occurs with a window of $1.6 \mathrm{sec}$ suggests both that the rules governing the relationships between electrical pattern between function related areas of the cortex are changed from time to time and also that passively conducted signals which would be invariant on time and best detected with maximum window size are not accounting for the changes that occur with changes in cognitive functioning.

\section{REFERENCES}

Bogen, J. E. The other side of the brain I, II, III. Bulletin of the Los Angeles Neurological Society, 1969, 34, 73-105, 135-162, 191-220.

Callaway, E., \& Harris, P. Coupling between cortical potentials from different areas. Science, 1974, 183, 873-875.

Elul, R. The genesis of the EEG. International Review of Neurobiology, 1972, 15, 227-272.

Galin, D., \& ORNSTEIN, R. Lateral specialization of cognitive mode: An EEG study. Psychophysiology, 1972, 9, 412-418.

GARNER, W. R. Uncertainty and structure as psychological concepts. New York: Wiley, 1962.

McGill, W. J. Multivariate information transmission. Psychometrika, 1954, 19, 97-116.

Shannon, C. E., \& WeAver, W. The mathematical theory of communication. Urbana: University of Illinois Press, 1949.

WINER, B. J. Statistical principles in experimental design. New York: McGraw-Hill, 1971. P. 49.

(Received for publication December 16, 1974; revision received July 14,1975 .) 